\title{
Magnetic resonance imaging of the head and spine: Effective for the clinician or the patient?
}

\author{
A K Dixon, J P Southern, A Teale, C E L Freer, L D Hall, A Williams, C Sims
}

\begin{abstract}
Objectives-To test how the results of magnetic resonance imaging influence clinicians' diagnoses and management plans for patients with cranial and spinal problems and to assess changes in the quality of life of these patients.

Design-Survey of patients undergoing cranial and spinal magnetic resonance imaging with questionnaires about diagnoses and intended management plans before and after imaging and quality of life questionnaires at the time of imaging and again four months later.
\end{abstract}

Setting-Regional magnetic resonance imaging and spectroscopy unit.

Subjects -100 consecutive patients referred for cranial imaging in early $1989 ; 100$ similar patients referred for spinal imaging.

Main outcome measures-Changes in clinicians' leading diagnoses after magnetic resonance imaging and their confidence in these diagnoses; changes in intended management plans; assessment of the contribution to the future management of the patient; changes in patients' quality of life.

Results-Magnetic resonance imaging altered the clinicians' leading diagnoses in 35 of 169 (21\%) cases. The clinicians became more confident about their leading diagnoses in 90 of $167(54 \%)$. There was a change in management plan in 113 of $182(62 \%)$. The clinicians considered that magnetic resonance imaging made an important contribution to management in 119 of $162(73 \%)$ patients. Overall, the patients' quality of life was unchanged at the four month assessment.

Conclusions-Magnetic resonance imaging of patients with cranial and spinal problems influences clinicians' diagnoses and management plans, but the quality of life of these patients remains unchanged.

Cambridge

A K Dixon, $\mathrm{MD}$, lecturer

Addenbrooke's Hospital, Cambridge CB2 $2 Q Q$

J P Southern, MFPHM, senior registrar, community medicine

A Teale, DCR, research

assistant

CE L Freer, FRCR, consultant radiologist

L D Hall, FRS, professor of medicinal chemistry

C Sims, DCR, superintendent radiographer

\section{Centre for Health}

Economics, University of

York, York YO1 5DD

A Williams, всом, professor

of economics

Correspondence to:

Dr A K Dixon, University

Department of Radiology,

Addenbrooke's Hospital,

Cambridge CB2 2QQ.

BMF 1991;302:78-82

\section{Introduction}

The development of new imaging tests is accompanied by a sequence of wonder, scepticism, and eventual acceptance similar to that associated with the introduction of a new drug. The evaluation of imaging tests, however, in terms of their efficacy, efficiency, and effectiveness is a necessary, if formidable, task..$^{1.3}$ Although much was learnt during the introduction of computed tomography, ${ }^{4.7}$ early work on the evaluation of magnetic resonance imaging mainly concerned efficacy and came under considerable criticism. ${ }^{8}$ More recent analyses have, in part, countered that criticism..$^{910}$ As part of our evaluation programme we audited certain aspects of the clinical use of magnetic resonance imaging to try to answer some of the questions posed by this new technique.

\section{Methods}

One hundred consecutive patients referred for cranial magnetic resonance imaging and 100 referred for spinal imaging formed the basis of the study. These constituted the NHS referrals to a regional unit in early familiar with this new technique. The study was approved by appropriate ethical committees.

\section{CLINICIANS}

Immediately the request for magnetic resonance imaging had been received a questionnaire was sent to the referring clinician (nearly always a consultant). Clinicians were asked to state their clinical diagnoses based on the history, examination, and investigations to date. They were also asked to indicate their confidence about each diagnosis on a scale of 0 to 10 $(10=$ certain $)$. Differential diagnoses were allowed - for example, a typical response might be: multiple sclerosis $6 / 10$, tumour $3 / 10$, others $1 / 10$. The diagnosis accorded the highest confidence was taken to be the leading diagnosis.

Clinicians also indicated their intended management plan for each patient. These were classified into four categories: further investigation (to obtain a diagnosis), reassurance only, symptomatic treatment (for chronic disorder), or curative treatment (surgery, etc).

When imaging had been performed (at a median of 25 days after the request form had been filled in) the report and films were sent back to clinicians along with the follow up questionnaire. This asked the same questions about diagnoses and management plans as before; clinicians had no recourse to the data on the original questionnaire at this stage. They were also asked to assess the contribution of the imaging result to future management (none, minor, moderate, or major contribution).

At the end of the study, changes in the leading diagnoses brought about by magnetic resonance imaging were noted. The patients' records were reviewed at six months to see whether there had been yet further changes in these diagnoses. Changes in clinicians' confidence about their leading diagnoses were also assessed, whether or not these diagnoses had altered as a result of imaging. These levels of confidence were compared by using Wilcoxon's signed rank test. " 1989, when both radiologists and clinicians had become
The 200 patients ranged in age from 9 months to 84 years (mean 44 years). Forty seven of the 100 referred for cranial imaging were men, as were 53 of those referred for spinal imaging. Among those referred for 
cranial imaging the most frequent clinical question was to confirm or exclude the presence of a space occupying lesion (35/100); possible multiple sclerosis (23), possible acoustic neuroma (21), and pituitary problems (15) were other common referrals. In those referred for spinal imaging possible disc herniation (35/100), stenosis (24), and tumour (13) were the most common referrals.

\section{INFORMATION FROM CLINICIANS}

The referring clinicians returned 182 sets of questionnaires; however, some of these were incomplete. Thus the numbers in the following groups differ.

The clinicians indicated an actual change in the leading diagnosis in 35 of the 169 patients $(21 \%)$ in whom full details about diagnoses were available (for example, case 1, box). Review of the case notes six months later showed only one further change in diagnosis: magnetic resonance imaging had suggested rheumatoid arthritis in the cervical spine but an epidural abscess was unexpectedly found at surgery.

The confidence of clinicians in their leading diagnoses before and after imaging is known for 167 patients. In 85 patients referred for cranial imaging confidence in leading diagnoses rose in $46(54 \%)$ (for example, case 2 , box), was unchanged in $33(39 \%)$, and fell in $6(7 \%)$; in 82 referred for spinal imaging confidence rose in $44(54 \%)$, was unchanged in 29 (35\%), and fell in $9(11 \%)$. These changes were significant $(z=6.5$ for cranial problems; $z=6 \cdot 1$ for

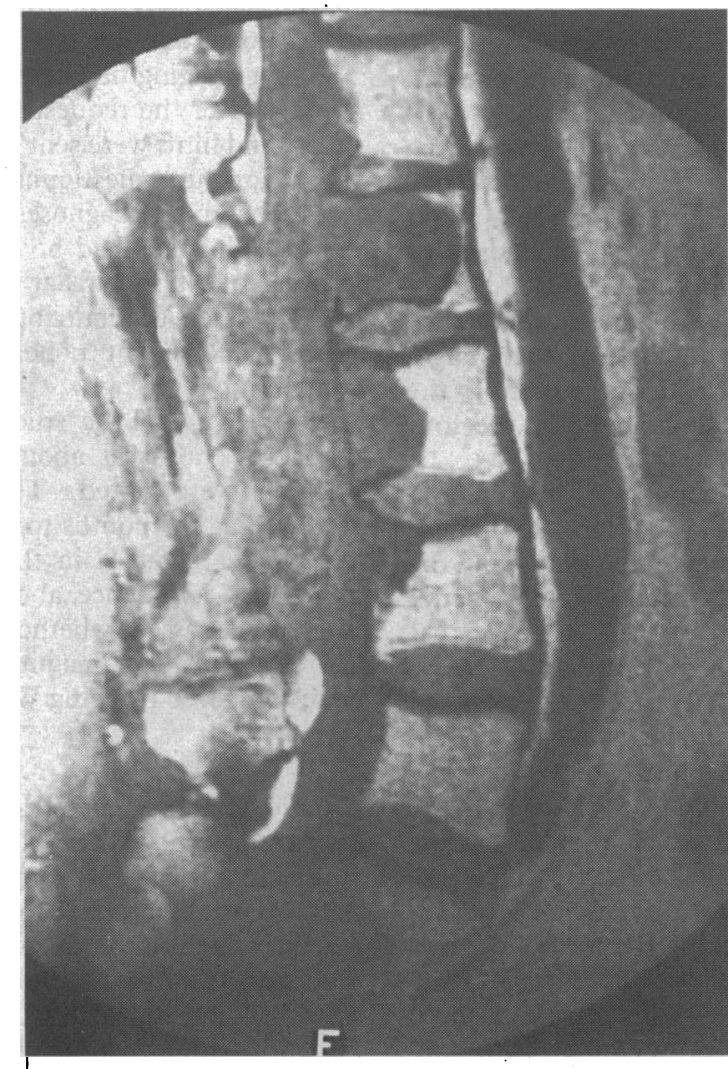

CASE 1 - a 52 year old patient had undergone surgery and radiation treatment for a spinal myxopapillary ependymoma three years before he was referred on account of deteriorating neurological signs. The leading diagnosis at referral was radiation myelopathy (confidence $9 / 10$; recurrent tumour $1 / 10$ ). This $\mathrm{T} 1$ weighted image shows a large expansile mass of low signal intensity occupying the lumbar canal and scalloping the posterior aspects of the vertebral bodies consistent with tumour recurrence. On the follow up questionnaire the clinician was confident $(10 / 10)$ that the diagnosis was that of recurrent tumour.

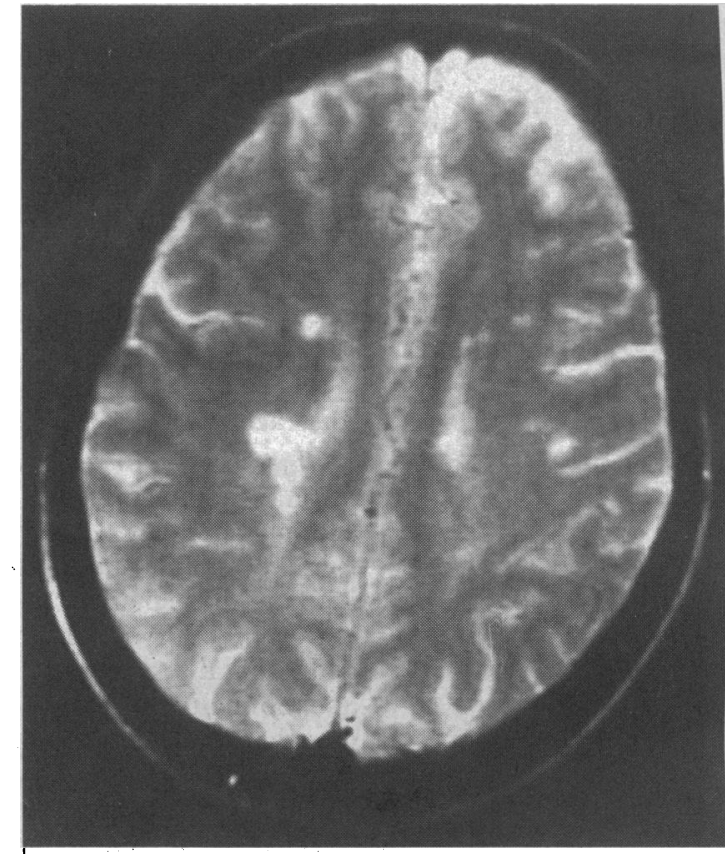

CASE $2-$ a 42 year old patient referred with a possible (5/10) diagnosis of multiple sclerosis. A functional disorder was the other possibility $(5 / 10)$. This T2 weighted image shows numerous areas of high signal intensity consistent with plaques of demyelination. At follow up the clinician was confident $(10 / 10)$ of the diagnosis of multiple sclerosis.

TABLE I-Clinicians' confidence in leading diagnosis before and after magnetic resonance imaging on scale from 0 to $10(10=$ certain diagnosis)

\begin{tabular}{lcccc}
\hline \multirow{5}{*}{$\begin{array}{l}\text { Before magnetic } \\
\text { resonance imaging }\end{array}$} & $0-5$ & $6-8$ & $9-10$ & Total No (\%) \\
\cline { 2 - 5 } & Referred for cranial imaging & $(n=85)$ \\
$0-5$ & 3 & 2 & 4 & $9(11)$ \\
$6-8$ & 1 & 8 & 26 & $35(41)$ \\
$9-10$ & 1 & 3 & 37 & $41(48)$ \\
\hline Total No(\%) & $5(6)$ & $13(15)$ & $67(79)$ & 85 \\
\hline \multicolumn{5}{c}{ After magnetic resonance imaging } \\
0-5 & Referred for spinal imaging $(n=82)$ \\
6-8 & 4 & 9 & 4 & $17(21)$ \\
$9-10$ & 2 & 16 & 16 & $34(41)$ \\
\hline Total No (\%) & 0 & 3 & 28 & $31(38)$ \\
\hline
\end{tabular}

spinal problems; $0.01<\mathrm{p}<0.05$ ). Table I shows the extent of these changes.

Table II shows the management plans before and after magnetic resonance imaging. In the 182 patients ( $93 \mathrm{head}, 89$ spine) for whom there was adequate data there was little correlation between management plans before and after imaging. The category of plan changed in 113 cases overall $(62 \%)$.

The clinicians commented on the contribution of the imaging result to future management in 162 patients. Among 85 referred for cranial imaging this contribution was described as major in $56(66 \%)$, moderate in 25 $(29 \%)$, and nil or minor in four $(5 \%)$. In the 77 referred for spinal imaging the contribution was classified as major in $63(82 \%)$, moderate in $11(14 \%)$, and nil or minor in three (4\%).

\section{INFORMATION FROM PATIENTS}

The initial quality of life questionnaires were given to 181 patients (inappropriate for 16 children and three colleagues). Forty patients chose not to return the questionnaires (five initially; 35 at follow up); in 11 patients the forms were incomplete. Thus there were 130 complete sets of questionnaires available for 
TABLE II-Clinicians' intended management plans before and after magnetic resonance imaging

\begin{tabular}{|c|c|c|c|c|c|}
\hline \multirow[b]{2}{*}{$\begin{array}{l}\text { Before magnetic } \\
\text { resonance imaging }\end{array}$} & \multicolumn{5}{|c|}{ After magnetic resonance imaging } \\
\hline & $\begin{array}{c}\text { Further } \\
\text { investigation }\end{array}$ & Reassurance only & $\begin{array}{l}\text { Symptomatic } \\
\text { treatment }\end{array}$ & $\begin{array}{l}\text { Curative } \\
\text { treatment }\end{array}$ & Total No $(\%)$ \\
\hline \multicolumn{6}{|c|}{ Referred for cranial imaging $(n=93)$} \\
\hline Further investigation & 22 & 16 & 23 & 7 & $68(73)$ \\
\hline Reassurance only & 0 & 3 & 0 & 1 & $4(4)$ \\
\hline Treatment of symptoms & 2 & 1 & 3 & 0 & $6(6)$ \\
\hline Curative treatment & 3 & 6 & 0 & 6 & $15(16)$ \\
\hline Total No (\%) & $27(29)$ & $26(28)$ & $26(28)$ & $14(15)$ & 93 \\
\hline \multicolumn{6}{|c|}{ Referred for spinal imaging $(n=89)$} \\
\hline Further investigation & 7 & 11 & 17 & 10 & $45(51)$ \\
\hline Reassurance only & 1 & 1 & 0 & 0 & $2(2)$ \\
\hline Treatment of symptoms & 0 & 1 & 5 & 1 & $7(8)$ \\
\hline Curative treatment & 3 & 6 & 4 & 22 & $35(39)$ \\
\hline Total No $(\%)$ & $11(12)$ & $19(21)$ & $26(29)$ & $33(37)$ & 89 \\
\hline
\end{tabular}

analysis (66 patients referred for cranial imaging, 64 referred for spinal imaging). Four patients who died before the follow up questionnaire were included in the study.

There was no overall change in the quality of life scores in these 130 patients over the four month period. For the 66 patients referred for cranial imaging the median score at referral was +0.986 (interquartile range +0.951 to +0.995$)$; this score was unchanged at follow up $(+0.986(+0.942$ to +1.000$))$. The median score for the spinal group (64 patients) was +0.942 $(+0.885$ to +0.956$)$ at referral and $+0.944(+0.870$ to $+0.972)$ at follow up.

\section{Discussion}

Evaluating new imaging tests is recognised to be a difficult task. ${ }^{214}$ Randomised controlled trials have been applied only for a few well defined clinical problems. ${ }^{45}$ Our audit on the opinions of the referring clinicians and the outcome for patients provides some measure of the effectiveness of magnetic resonance imaging in routine clinical practice. Such evaluations have not often been undertaken. ${ }^{16}$

The fact that the magnetic resonance imaging findings affected clinicians' leading diagnoses suggests that the test is quite widely accepted. This acceptance is confirmed by the high confidence in these diagnoses after imaging ( 115 of 169 scored $9 / 10$ or $10 / 10$ confident). There was virtually no departure from these leading clinical diagnoses on review of the case notes at six months, which lends support to the credibility of the test.

The few changes in leading diagnoses and the high confidence in this diagnosis even before magnetic resonance imaging (median score 8/10) probably reflects the experience of the referring clinicians. In addition, many patients had already been partially evaluated by other techniques. These results suggest that we should try harder to select referrals where real diagnostic uncertainty exists and to encourage magnetic resonance imaging early on in the sequence of diagnostic tests for those clinical problems where it is of particular value.

The comparison of clinicians' management plans before and after imaging is one of the most interesting features of this study (table II). At first glance the proportion of patients considered suitable for curative treatment (usually surgery) within both the cranial and spinal groups seems similar before and after imaging (cranial: 15 before, 12 after; spinal: 35 before, 33 after), but only some of these patients (6 of 12 cranial and 22 of 33 spinal) were considered for curative treatment both before and after the imaging result. Of those patients who were originally destined for an operation, surgery was ultimately considered appropriate in only six of the 15 patients in the cranial group and in 22 of 35 in the spinal group. Further analysis of the 113 changes in management plans showed that information from the test generally moved the patient towards definitive treatment (whether curative or treatment of symptoms or only reassurance) and away from further investigation.

The fact that we only have adequate information from the clinicians on only about $85 \%$ of patients raises a potential criticism. It may be unrealistic, however, to hope for much better voluntary compliance. Similarly, the rate for adequate completion of both of the quality of life questionnaires $(130 / 181,72 \%)$ is in keeping with other studies on outcome.

It may seem disappointing that investigation by magnetic resonance imaging was not accompanied by any improvement in quality of life, but there are several reasons why this did not occur. Most of these patients had chronic conditions, which might not change over the study period. Even for a condition that might be influenced by surgery an improvement may be delayed until after the immediate postoperative effects have subsided. Furthermore, residual disability is to be expected after some successful operations. In conditions such as multiple sclerosis that have an intermittent course the quality of life will be affected by the disease process rather than by any benefits from the test. It is of interest that the greatest changes in quality of life were in a negative direction; if the four deaths and one patient assessed in the immediate postoperative period had been excluded, the overall change would have been slightly positive. Also without a control group we do not know how the patients would have fared without magnetic resonance imaging. The steady quality of life might represent a significant benefit, particularly for those patients in whom the result had led to a change in management plan. More randomised trials (such as that by Teasdale $e t a l^{15}$ ) will provide further information.

Cooper et al criticised attempts at evaluating magnetic resonance imaging, ${ }^{8}$ and many of their criticisms are valid. ${ }^{17}$ But the overriding point is that assessing any new form of technology in medicine particularly new methods of imaging, is extremely difficult. Some of the criticisms of Cooper et al could be levelled against the results of our study but not that we ignored the opinions of referring clinicians or the outcome for the patients. ${ }^{18}$ As far as imaging tests are concerned, the referring clinician is the ultimate "consumer," perhaps more so than the patient. Thus whether a new imaging test assists the clinician's understanding and management of the patient is the paramount arbiter as to whether the new test is helpful or not. In this respect magnetic resonance imaging does well; perhaps too well if judged by the current waiting lists for this form of imaging in Britain. Although their clinical condition may not be improved through the use of magnetic resonance imaging, patients can be assured that they have had an efficacious test and that their clinician is, in general, more confident about the diagnosis upon which to base subsequent management.

This work would not have been possible without the generosity of the Addenbrooke's Hospital Cancer Scanner Appeal Fund, the East Anglian Regional Health Authority, and the Department of Health. The Addenbrooke's site has a research agreement with Picker International.

We thank our clinical colleagues: clinicians who referred the patients and completed the questionnaires and nurses and radiographers who discussed the project with the patients. Professor T Sherwood gave helpful advice and encouragement and Drs A T Moore, D Rubenstein, D Rhys Williams, and I Wilkinson helped with evaluation, as have radiologists in several general hospitals in East Anglia, who facilitated the collection of follow up data.

1 Dollery C. The end of the age of optimism. London: Nuffield Provincial Hospitals Trust, 1978:59. (Rock Carling Fellowship.) 
2 Hillman BJ. Evaluating magnetic resonance: what do we need to know? Invest Radiol 1986;21:289-9

3 St Leger AS, Allen D, Rowsell KV. Procedures for evaluating innovatory proposals. $B M f$ 1989;299:1017-8

4 Dixon AK, Kelsey FI, Kingham JGC, McLean AM, White FE. Computed tomography in patients with an abdominal mass: effective and efficient? A controlled trial. Lancet 1981;i:1199-201.

5 Russell I, Williams A. Evaluation of computerised tomography: a review of research methods. In: Culyer AJ, Horisberger B, eds. Economic and medical evaluation of health care techniques. Berlin: Springer Verlag, 1983:298-379.

6 Anonvmous. Body computed tomography: wanted or needed? [Editorial]. Lancet 1984;ii:961-2.

Morre AT, Dixon AK, Rubenstein D, Wheeler T. Cost benefit evaluation of body computed tomography. Health Trends 1987;19:8-12.

8 Cooper LS, Chalmers TC, McCally M, Berrier J, Sacks HS. The poor quality of early evaluations of magnetic resonance imaging. fAMA 1988;259: 3277-80.

9 Shuman WP. The poor quality of early evaluations of MR imaging: a reply A7R 1988:151:857-8.

10 Hillman $B J$. The assessment of $M R$ imaging. $A 7 R$ 1988:151:858-60.
11 Siegel S, Castellau NJ. Nonparametric statistics for the behavioural sciences. New Fork: McGraw-Hill, 1988:298-303.

12 Gudex C, Kind P. The QALY toolkit. York: Centre for Health Economics, University of York, 1988. (Discussion paper No 38 .

13 Kind P, Rosser R, Williams A. Valuation of quality of life: some psychometric evidence. In: Jones-Lee MW, ed. The value of life and safety. Amsterdam North-Holland, 1982:159-70.

14 Anonymous. The value of diagnostic tests [Editorial]. Lancet 1979;i:809-10.

5 Teasdale GM, Hadley DM, Lawrence A, et al. Comparison of magnetic resonance imaging and computed tomography in suspected lesions in the posterior cranial fossa. BMF 1989;299:349-55.

16 Franken EA, Berbaum KS, Dunn V, et al. Impact of MR imaging on clinical diagnosis and management: a prospective study. Radiology 1986;161: $377-80$.

17 Kent DL, Larson EB. Magnetic resonance imaging of the brain and spine -is clinical efficacy established after the first decade? Ann Intern Med 1988;108: $402-24$

18 Greenfield $S$. The state of outcome research: are we on target? $N$ Engl 7 . Med 1989;320:1142-3.

(Accepted 1 November 1990)
Stobhill Hospital, Glasgow J R Smith, MRCOG, research fellow, department of gynaecology

J Murdoch, MD, senior registrar in gynaecology A J Dougall, MRCPATH consultant bacteriologist C A Forrest, MRCOG, consultant gynaecologist J A Davis, FRCOG, consultant gynaecologist

\section{Maryhill Health Centre,}

H MacKinnon, FRCGP, general practitioner

D Baillie, MRCGP, general practitioner

D M Byford, MRCGP, general practitioner

Royal Infirmary, Glasgow

D Carrington, MRCPATH,

consultant virologist

C E Frew, BSC, senior

virologist

Correspondence to:

Mr J R Smith, The Jefferis

Wing, St Mary's Hospital,

London W2.

BMF 1991;302:82-4

\title{
Prevalence of Chlamydia trachomatis infection in women having cervical smear tests
}

\author{
J R Smith, J Murdoch, D Carrington, C E Frew, A J Dougall, H MacKinnon, D Baillie, D M Byford, \\ C A Forrest, J A Davis
}

\section{Abstract}

Objective-To determine the prevalence of sexually transmitted diseases in patients with normal and abnormal cervical smears.

Design-A prospective study of asymptomatic women with normal cervical smears attending their general practitioner and newly referred patients with abnormal smears attending a colposcopy clinic.

Setting-A hospital based colposcopy clinic and an urban general practice (list size 5500) in north west Glasgow.

Subjects -197 asymptomatic women attending their general practitioner for cervical smear tests and 101 randomly selected patients attending the colposcopy clinic for investigation of abnormal smears

Main outcome measures-Presence of various sexually transmitted infections as determined by culture and serological tests.

Results-Of the 101 women with cytological abnormalities, six had current chlamydial infection proved by culture and none had gonococcal infection; of the 197 women with normal smears, $24(12 \%)$ had a chlamydial infection and two had gonorrhoea. Serological studies for Chlamydia trachomatis specific antibody also indicated that a large proportion of patients had been exposed to this agent in both groups. There was no significant difference between the groups in the prevalence of any sexually transmitted disease studied.

Conclusion-A high prevalence of chlamydial infection is present in women in north west Glasgow irrespective of their cervical cytological state.

\section{Introduction}

The prevalence of Chlamydia trachomatis, Neisseria gonorrhoeae, herpes simplex virus, Candida albicans, and Trichomonas vaginalis infections in women varies with the clinical setting. In previous studies of asymptomatic women in general practice the prevalences of $C$ trachomatis and $N$ gonorrhoeae were $10.7 \%^{1}$ and $0.9 \%^{2}$ respectively, and prevalences of $3-8 \%^{34}$ and $1 \cdot 8 \%$ respectively were recorded in hospital gynaecology practice. Controversy surrounds the prevalence of chlamydial infection as highly variable rates have been measured in different studies. Screening tests for chlamydia have not been readily available to genera practitioners, and many doctors are not aware of the local prevalence rate. This is an important factor when considering whether to screen asymptomatic patients.

Cervical intraepithelial neoplasia is associated with exposure to multiple sexual contacts, a feature which also predisposes to the acquisition of sexually transmitted diseases. ${ }^{6}$ No study has compared the prevalence of sexually transmitted diseases in patients attending a colposcopy clinic for cytological abnormalities with that in patients in general practice from the same area whose smears are normal. We report such a study in north west Glasgow.

Patients and methods

REFERRALS TO COLPOSCOPY CLINIC

One hundred and one patients attending the colposcopy clinic at Stobhill Hospital were enrolled over one year. All were a subsample of consecutive new referrals selected at random. They had abnormal cytological findings as categorised by Papanicolaou class II with atypia (borderline nuclear abnormality; 20 cases) class III (mild to moderate dyskaryosis; 72 cases), class IV (severe dyskaryosis; eight cases), or class V (malignant cells; one case). All socioeconomic classes were represented, but most patients were in social class III. The age range of the patients was 19-58 years (mean 30)

At the initial visit full microbiological investigation was performed for $C$ trachomatis, $N$ gonorrhoeae, $T$ vaginalis, $C$ albicans, and herpes simplex virus. Swabs taken from the urethra, endocervix, and rectum were directly plated on to Thayer's medium for culture of $N$ gonorrhoeae and the plates incubated at $37^{\circ} \mathrm{C}$ in a carbon dioxide enriched atmosphere for 48 hours. Cervical smears were obtained using an Ayres spatula. Tests for $C$ trachomatis were performed by culture in McCoy cells on swab samples obtained from the endocervix. Positive cultures were identified when monoclonal antibodies (Microtrak-SYVA, United Kingdom) bound successfully to chlamydial inclusions.

In addition, blood was drawn for serological tests for chlamydia. Microimmunofluorescence tests were performed for $C$ trachomatis strains $\mathrm{D}-\mathrm{K}$ by using an antigen pool (IOL P2). Reciprocal antibody titres of $C$ trachomatis specific IgG and IgM were evaluated for each sample and interpreted as: IgG titre $<16$, probably no previous exposure to a chlamydial agent; IgG titre $>128$, suggestive evidence of recent infection; IgG 